\title{
Antimicrobial stewardship programmes: bedside rationing by another name?
}

\author{
Simon Oczkowski
}

Correspondence to Dr Simon Oczkowski, McMaster University, Juravinski Hospital Room A3-20, 711 Concession St., Hamilton, ON, Canada L8V 1C1; oczkowsj@mcmaster.ca

Received 13 March 2015 Revised 17 January 2017 Accepted 13 February 2017 Published Online First 15 March 2017

\section{SLinked}

http://dx.doi.org/10.1136/ medethics-2017-104572

\section{CrossMark}

To cite: Oczkowski S.

$J$ Med Ethics

2017;43:684-687.

\section{ABSTRACT}

Antimicrobial therapy is a cornerstone of therapy in critically ill patients; however, the wide use of antibiotics has resulted in increased antimicrobial resistance and outbreaks of resistant disease. To counter this, many hospitals have instituted antimicrobial stewardship programmes as a way to reduce the inappropriate use of antibiotics. However, uptake of antimicrobial stewardship programmes has been variable, as many clinicians fear that they may put individual patients at risk of treatment failure. In this paper, I argue that antimicrobial stewardship programmes are indeed a form of bedside rationing, and explore the risks and benefits of such programmes for individual patients in the intensive care unit, and the critically ill population in general. Using Norman Daniels' Accountability for Reasonableness as a framework for evaluating resource allocation policies, I conclude that antimicrobial stewardship programmes are an ethically sound form of bedside rationing.

\section{INTRODUCTION}

Infectious diseases are one of the leading causes of admission and death in the intensive care unit (ICU). ${ }^{1}$ The cornerstone of therapy for all such cases is the use of antimicrobial agents. The 'spectrum' of antimicrobial agents refers to the number of different organisms against which the drug is effective, with 'broad-spectrum' antimicrobials having efficacy against many infective organisms, and 'narrow-spectrum' antimicrobials only being effective against a few. In the sickest patients, the time until administration of an antibiotic which treats the organism causing the infection is associated with up to a $7.6 \%$ absolute increase in mortality per hour. ${ }^{2}$ Hence, the early initiation of broad-spectrum antibiotics is considered the standard of care, and received a 'strong' recommendation in the 2012 Surviving Sepsis Guidelines. ${ }^{3}$

At the same time, the use of broad-spectrum antibiotics is associated with the development of antibiotic-resistant organisms (AROs), which due to their resistance properties are associated with higher healthcare costs as well as increased morbidity and mortality compared with non-AROs. ${ }^{4}$ In order to combat this growing problem, the Infectious Diseases Society of America (IDSA) released guidelines for developing antimicrobial stewardship programmes (ASPs), which aim to prevent the development of AROs by narrowing the spectrum, reducing the dose and shortening the duration of antimicrobials used. Although their goal is to '...lead to the best clinical outcome for the treatment or prevention of infection while producing the fewest possible side effects and the lowest risk for subsequent resistance, ${ }^{5}$ clinicians often have concerns that ASPs ration care, putting their patients at risk of worsening infection due to hasty discontinuation or narrowing of antibiotics. ${ }^{6}$ In part for this reason, uptake of ASPs has been variable, with recent surveys of American hospitals finding that only half have a formal ASP. ${ }^{7}$

In this paper, I will argue that despite the claims of proponents, ASPs are in fact a form of bedside rationing, which despite posing some risk to individual patients, can reduce the incidence of AROs and lower healthcare costs. Characterising ASPs as a form of rationing is not a criticism. Rather, I argue that ASPs are consistent with Norman Daniels' model of 'accountability for reasonableness' (AFR) and are thus a fair and ethically sound form of bedside rationing. ${ }^{9}$

\section{ARE ASPS A FORM OF BEDSIDE RATIONING?}

The first claim to be proved is that ASPs are in fact a form of bedside rationing. I adopt the definition of bedside rationing from Ubel and Goold, which includes three criteria: ${ }^{10}$

1. Clinicians must withhold, withdraw or fail to recommend a service that, in their clinical judgment, is in the patient's best medical interests.

2. They must do so primarily to promote the interests of someone other than the individual patient.

3. The clinician must have control over the use of the medically beneficial service.

The first condition is required for an action to be considered an act of rationing: an individual patient's immediate interests must be compromised. The second and third criteria are required for such rationing to be considered of the 'bedside' variety, as opposed to a form of rationing imposed on clinicians by external forces. I address each of these three criteria in turn.

To meet the first criterion requires the demonstration that ASPs result in the withholding or withdrawal of care that is in the patient's best medical interest. For critically ill patients, failure to initiate appropriate broad-spectrum antibiotics is associated with an increase in mortality. But even in the most severe infections, a positive culture result indicating which organism is causing the infection occurs in less than two-thirds of cases. ${ }^{11}$ In the remainder, the use of antimicrobials remains 'empirical', that is, the 'best guess' of which organism is causing the illness. By narrowing the spectrum and decreasing the dose or duration of antimicrobial therapy, ASPs theoretically put individual patients, especially 
those being treated empirically, at risk of treatment failure or infectious relapse. Although all patients, including those currently receiving antibiotics, may benefit in the long term from the continued effectiveness of antimicrobials, this assumes the individual will survive long enough to enjoy this benefit. Although critically ill patients are generally too sick to make their immediate interests known, it is reasonable to assume that for most people, an increased likelihood of short-term survival would likely be of higher priority than theoretical future benefits of antimicrobial effectiveness. Thus, for the clinicians and families making decisions on the patient's behalf, when ASPs recommend that a physician narrows the spectrum or shortens the duration of antibiotics, this seems to be rationing, on balance, against the patient's immediate best interests, thereby fulfilling criterion. ${ }^{1}$ Further emphasising the concept of ASPs as rationing is the recognition of physician concerns about rationing as a potential barrier to the implementation of ASPs. ${ }^{12}$ However, as I note below, this rationing may be ethically justified.

The second two criteria are more straightforward to demonstrate. ASPs have been shown to result in lower costs for hospitals $^{13} 14$ and to result in lower rates of AROs, ${ }^{14}{ }^{15}$ both of these are clearly of benefit for hospitals, and future patients. Neither directly promotes the interests of the individual patient whose care is being managed by the ASP (although if the patient is fortunate enough to survive the current illness, and unfortunate enough to get another infection, he/she stands a chance of benefitting from reduced rates of AROs). Finally, as to the third criterion, ASPs are distinct from other forms of antimicrobial rationing because they are meant to be a part of the bedside decision-making of individual clinicians. According to the IDSA guidelines, the first core strategy of ASPs is the 'audit of antimicrobial use with direct interaction and feedback to the prescriber, performed by either an ID physician or a clinical pharmacist with infectious diseases training'. The second core strategy is formulary preauthorisation, where the approval of the ID physician or pharmacist is required to gain access to an antimicrobial. In both cases, the treating physicians jointly have the discretion to prescribe or limit the therapies used, and decide in what cases rationing is to be used, thus fulfilling the third criterion.

In summary, the core strategies of ASPs are a form of bedside rationing, where clinicians limit the antimicrobials that are used, in order to lower costs and prevent the development of AROs. Although many clinicians are wary of bedside rationing, it has been argued that such rationing is a necessary part of modern healthcare. ${ }^{16}$ The progressive acceptance of ASPs since their introduction in the $1980 \mathrm{~s}^{5}$ may be due to the fact that despite clinician reluctance to participate in bedside rationing, ASPs are a fair, ethically sound example of the practice. In the next section, I will explore how ASPs meet many of the ideal qualities of healthcare rationing.

\section{A MODEL FOR RATIONING HEALTHCARE RESOURCES}

One of the most widely accepted models for healthcare rationing is that described by Norman Daniels, called 'AFR', the premise of which is that the moral legitimacy of healthcare rationing decisions depends on the process that is used to set them as much as the identity of the decision-maker or the rationing outcomes. ${ }^{9}$ According to AFR, there are four requirements for a rationing process to be considered fair: ${ }^{17}$

1. Publicity condition: decisions and rationales must be publicly accessible.
2. Relevance condition: must appeal to reasons and principles which can be generally agreed on are relevant.

3. Appeals/revision condition: provision of a mechanism for dispute resolution or revision of decision.

4. Enforcement condition: regulation of the process to ensure that criteria 1-3 are met.

As laid out in the IDSA guidelines, ASPs fulfil all of the AFR criteria, and can thereby serve as a model bedside rationing. I will address each criterion below.

\section{Publicity condition}

By making the rationing decision and its rationale available for scrutiny, the publicity condition can help to make the rationing process fairer by insuring that inconsistencies in rationing can be caught. ${ }^{18}{ }^{19}$ In the context of ASPs, a major question is who the 'public' should be? ICU patients are often too sick to be involved in their own decision-making, and complex decisions such as antimicrobial choice are generally made by the health care provider (HCP), with minor input from the surrogate decision maker (SDM), in a shared decision-making model on the patient's behalf. ${ }^{20}$ In instances where the wishes of a patient are unknown, HCPs and SDMs must still act in accordance with a patient's best interests, and generally do so through a Rawlsian 'veil of ignorance'-they attempt to make the decision which a rational, moral person would make, not knowing if they would have to live with the consequences of the decision. ${ }^{21}$ In either case, the HCP is the avatar for the patient's interest, hence I would argue that the 'publicity condition' in ASPs refers to the HCPs involved in the circle of care of a patient, rather than the patient himself or herself.

The IDSA guidelines suggest that the prospective audit of antimicrobial use should be '.. with direct interaction and feedback to the prescriber' (p. 164). Furthermore, education about the rationale for the antimicrobial decision is '.... an essential element of any programme and can provide a foundation of knowledge that will enhance and increase the acceptance of stewardship strategies' (p. 166). Even the second core component of ASPs, formulary preauthorisation, requires effective educational strategies to be effective, so that clinicians do not just 'squeeze the balloon' and make poor alternative antimicrobial choices which lead to the development of novel AROs. ${ }^{22}$ Publicity is therefore a recommended component of ASPs, and the one required for their continued efficacy.

\section{Relevance condition}

The condition of relevance requires that decisions about rationing be made using values and principles that are '....accepted as relevant by people who are disposed to finding mutually justifiable terms of cooperation' (ref. 17, p. 329). As noted above, the input of the patients themselves is generally limited by the circumstances of their incapacitating illness. In the absence of input of the values and principles of the patients themselves, a fair decision to ration can still be made by people making decisions through a Rawlsian 'veil of ignorance', as if they were unaware whether they personally would have to live with the consequences of that decision. Disagreement with regard to ASPs take place among generally agreed-on principles and values, including the prevention of the development of AROs, reduced healthcare costs and maximising of clinical benefit for individual patients. ${ }^{5}$ Fortunately, few clinicians, patients or policy makers would debate the worthiness of these goals consistent with those that would be used under a 'veil of ignorance'. The major conflict in bedside rationing is balancing the interests of an individual patient with those of society in general. This is 
a situation of reasonable disagreement, as opposed to mere disagreement, and thus in keeping with the relevance condition. ${ }^{19}$

\section{Appeals condition}

The appeals condition requires that there be a mechanism in place by which one can appeal or request a revision of the rationing decision in question. For clinicians, this is the requirement that in any given antimicrobial rationing, there is an opportunity to revise or overrule the rationing if the patient is considered to be an exceptional case. The IDSA guidelines state that although ASPs are broadly applicable to all hospitalised patients, including ICU patients, they are 'not a substitute for clinical judgment, and clinical discretion is required in the application of guidelines to individual patients'. Furthermore, the guidelines recommend that ASPs receive approval from relevant medical staff to ensure acceptance and support (p. 163). In part, this is because for an ASP to be effective, it must respond to concerns from clinicians taking care of individual patients, or risk having such clinicians 'gaming' the system and undermining any potential benefits of the programme. ${ }^{22}{ }^{23}$ Hence, the appeals condition is actually required for ASPs to be an effective form of rationing.

\section{Enforcement condition}

The enforcement condition is met in the IDSA guidelines by the use of a multidisciplinary team, who either provides audit and feedback of antimicrobial choices or a formulary pre-approval. According to the guidelines, the ideal makeup of the team includes 'a clinical microbiologist, an information system specialist, an infection control professional and hospital epidemiologist', who are selected for their ability to interact effectively with clinicians, helping to make the purpose behind rationing decisions clear, providing suggestions for antimicrobial changes (but also willing to allow such suggestions to be overruled where appropriate) and ensuring that the relevant concerns are met through the use of clinical informatics and epidemiology. ${ }^{5}$ This 'package' of experts allows for the enforcement of the first three AFR criteria.

\section{ARE ASPS AN EFFECTIVE FORM OF RATIONING?}

The AFR criteria are not merely conditions for fair rationing, but they also prerequisite for an ASP to be a clinically effective form of bedside rationing. An ASP which is not public in its decisions, does not value the relevance of individual patient care, and provides no mechanism for clinicians to appeal rationing decisions on behalf of individual patients is unlikely to succeed, as clinicians 'game' the system. Ubel identifies this concern as one of the reasons why rationing decisions must be made at the bedside rather than be imposed on clinicians. ${ }^{16}$ Hence, the real test of whether or not the AFR criteria are effectively and appropriately met can be determined in part by determining whether ASPs effectively meet their desired clinical goals. For instance, an ASP where the HCPs refuse to limit antimicrobials and increasing rates of AROs signifies that the process itself may not be perceived by HCPs as fair and its methods re-evaluated. Perhaps the HCPs require education on the long-term benefits of the ASP, or a clearer appeals mechanism to decide when to not restrict antibiotics. For these reasons, I would argue that other incentives (financial, administrative, etc) not be routinely used to encourage HCPs or ASPs to meet targets. The potential for conflict of interest in bedside rationing is too great, and the stakes too high to further conflate the interests of the HCP and the patient for whom decisions are being made. Such incentives effectively destroy the 'veil of ignorance' and violate the relevance criterion-remuneration of HCPs is not relevant to the interests of the patient.

Thankfully, there have been many studies of the implementation of ASPs which can address concerns about their use. ${ }^{24}$ ASPs appear to be effective in reducing costs in the long term ${ }^{13}$ and in preventing the development of AROs without resulting in worse clinical outcomes. ${ }^{14}$ This success has been shown to translate into multiple centres, ${ }^{14} 1523$ and ASPs are gradually becoming part of the standard of care in ICUs. ${ }^{6}$ Hence, ASPs are likely one of the most effective models of bedside rationing, demonstrating both fair process as described by AFR, and excellent outcomes as a result of well-monitored rationing, with a minimal impact on the care of individual patients. Potential compromise of individual patient care in the short term can pay off over time with improved outcomes for the critically ill population in general.

\section{REJECTING ASPS AS A FORM OF BEDSIDE RATIONING}

There are a few concerns some may have with this interpretation of ASPs. Some may dispute that ASPs are really a form of bedside rationing at all, given that the emerging data from ASPs finds that patient outcomes are no worse following the implementation of an ASP-according to Ubel's first criterion, bedside rationing must be a decision to withhold or withdraw care that is in an individual patient's best interest. In fact, in some cases ASPs have resulted in improvements in patient outcomes, such as a lower risk of Clostridium difficile infections secondary to inappropriate antibiotic use. ${ }^{25}{ }^{26}$ However, we must distinguish between the effects of ASPs on populations of critically ill patients and on individual critically ill patients. The purpose of ASPs is to improve the health of the entire critically ill population. The failure to demonstrate such improvements would be a failure of ASPs. The benefits ASP demonstrate for the health of populations does not diminish the risk that they pose to individual patients within that population-they are the ones who carry the burden of risk, by receiving a shorter dose or narrow-spectrum antibiotic with uncertain effects on their personal health-such patients need to survive their initial illness before they could possibly benefit from the reduced risk of AROs or secondary infections which ASPs can provide. Hence, ASPs withhold or withdraw care that is in the individual patient's best interest and puts them at short-term risk, although there is a theoretical long-term benefit if those risks are well managed and the patient survives. The success of ASPs does not discredit the claim that they are a form of bedside rationing, but rather indicates that they are a successful form of bedside rationing.

\section{CONCLUSIONS}

ASPs meet all of the criteria for bedside rationing as defined by Ubel and Goold. The IDSA guidelines for the implementation of ASPs also meet the requirements of AFR as described by Daniels. The growing success of ASPs therefore demonstrates a successful implementation of both bedside rationing and the principles of AFR, and is an exemplary demonstration of how bedside rationing can be procedurally fair and lead to excellent clinical outcomes. The short-term compromise ASPs impose on the care of individual critically ill patients results in improved outcomes for the critically ill population in general.

Twitter Follow Simon Oczkowski @simon_ocz

Acknowledgements The author would like to thank Dr Jennifer Gibson for her critical review and editing of the manuscript.

Competing interests None declared.

Provenance and peer review Not commissioned; externally peer reviewed. 


\section{REFERENCES}

1 Braber A, van Zanten AR. Unravelling post-ICU mortality: predictors and causes of death. Eur J Anaesthesiol 2010;27:486-90.

2 Kumar A, Roberts D, Wood KE, et al. Duration of hypotension before initiation of effective antimicrobial therapy is the critical determinant of survival in human septic shock. Crit Care Med 2006;34:1589-96.

3 Dellinger RP, Levy MM, Rhodes A, et al. Surviving sepsis campaign: International Guidelines for Management of Severe Sepsis and Septic Shock: 2012. Crit Care Med 2013;41:580-637

4 Cosgrove SE. The relationship between antimicrobial resistance and patient outcomes: mortality, length of hospital stay, and health care costs. Clin Infect Dis 2006:42(Suppl 2):S82-9.

5 Dellit TH, Owens RC, McGowan JE, et al. Infectious Diseases Society of America and the Society for Healthcare Epidemiology of America Guidelines for Developing an Institutional Program to Enhance Antimicrobial Stewardship. Clin Infect Dis 2007:44:159-77.

6 George P, Morris AM. Pro/con debate: Should antimicrobial stewardship programs be adopted universally in the intensive care unit? Crit Care 2010;14:205.

7 Doron S, Nadkarni L, Lyn Price L, et al. A Nationwide survey of antimicrobia stewardship practices. Clin Ther 2013;35:758-65.e20.

8 Duane TM, Zuo JX, Wolfe LG, et al. Surgeons do not listen: evaluation of compliance with antimicrobial stewardship program recommendations. Am Surg 2013:79:1269-72.

9 Daniels N. Accountability for reasonableness: establishing a fair process for priority setting is easier than agreeing on principles. BMJ 2000;321:1300-1.

10 Ubel PA, Goold S. Recognizing bedside rationing: clear cases and tough calls. Ann Intern Med 1997:126:74-80.

11 Angus DC, Linde-Zwirble WT, Lidicker J, et al. Epidemiology of severe sepsis in the United States: analysis of incidence, outcome, and associated costs of care. Crit Care Med 2001;29:1303.

12 Sunenshine $\mathrm{RH}$, Liedtke LA, Jernigan DB, et al. Role of infectious diseases consultants in management of antimicrobial use in hospitals. Clin Infect Dis 2004;38:934-8.
13 Beardsley JR, Williamson JC, Johnson JW, et al. Show me the money: long-term financial impact of an antimicrobial stewardship program. Infect Control Hosp Epidemiol 2012;33:398-400

14 Kaki R, Elligsen $M$, Walker $S$, et al. Impact of antimicrobial stewardship in critical care: a systematic review. J Antimicrob Chemother 2011;66:1223-30.

15 Hurford A, Morris AM, Fisman DN, et al. Linking antimicrobial prescribing to antimicrobial resistance in the ICU: before and after an antimicrobial stewardship program. Epidemics 2012;4:203-10.

16 Ubel PA. Physicians, thou shalt ration: the necessary role of bedside rationing in controlling healthcare costs. Healthc Pap 2001;2:10-21.

17 Daniels N, Sabin J. Limits to health care: fair procedures, democratic deliberation, and the legitimacy problem for insurers. Philos Public Aff 1997;26:303-50.

18 Daniels N. Just health: meeting health needs fairly. Cambridge: Cambridge University Press, 2008.

19 Rid A. Justice and procedure: how does "accountability for reasonableness" result in fair limit-setting decisions? J Med Ethics 2009;35:12-16.

20 Légaré $F$, Labrecque $M$, LeBlanc $A$, et al. Training family physicians in shared decision making for the use of antibiotics for acute respiratory infections: a pilot clustered randomized controlled trial. Health Expect 2011;14(Suppl 1):96-110.

21 Rawls J. A theory of justice (paperback edition). Oxford: Oxford University Press, 1971.

22 Burke JP. Antibiotic resistance-squeezing the balloon? JAMA 1998;280:1270.

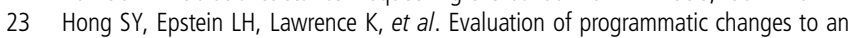
antimicrobial stewardship program with house officer feedback. J Eval Clin Pract 2013;19:388-92

24 Guervil DJ, Klinker KP, Reardon NT, et al. Measuring quality metrics to identify and monitor antimicrobial stewardship program quality improvement efforts. Infect Control Hosp Epidemiol 2014;35:101-3.

25 Leung V, Gill S, Sauve J, et al. Growing a "positive culture" of antimicrobial stewardship in a community hospital. Can J Hosp Pharm 2011;64:314-20.

26 Owens RC Jr, Fraser GL, Stogsdill P. Antimicrobial stewardship programs as a means to optimize antimicrobial use. Insights from the Society of Infectious Diseases Pharmacists. Pharmacotherapy 2004;24:896-908. 